\title{
Non-Cartesian Frame Transformation-Based Control of a Three-Phase Power Converter During Unbalanced Voltage Dip - Part II: Simulation And Experiment
}

\author{
Grzegorz Iwański, Paweł Maciejewski, Tomasz Łuszczyk
}

Warsaw University of Technology, Institute of Control and Industrial Electronics, ul. Koszykowa 75, 00-662 Warszawa, Poland

Received March 04, 2019; Accepted July 09, 2019

Abstract: The paper presents a control method for the three-phase power converter operating under unbalanced grid voltage conditions. The method uses a new transformation to the non-Cartesian frame, which makes the controlled current vector components balanced in this frame even if originally the three-phase current is referenced as unbalanced. Furthermore, Park's transformation makes the controlled variables constant, which allows to apply proportional-integral terms as current controllers independent of the required control target. Several control targets known from literature have been analyzed with regard to the required new transformation parameters, and the transformation parameters for all targets have been found. Simulation results are shown to prove the theoretical analysis, and the experimental test results are presented as practical validation of the proposed use of the non-Cartesian frame in control.

Keywords: $A C-D C$ power conversion $\bullet$ current control $\bullet$ current limitation

\section{Introduction}

The grid-connected three-phase power converter presented in Fig. 1 operating under unbalanced grid voltage conditions can be controlled with different current targets, such as symmetrical current (Saccomando and Svensson, 2001), unbalanced current with asymmetry corresponding to grid voltage asymmetry (Castilla et al., 2014) and unbalanced current with asymmetry opposite to grid voltage asymmetry (Hang and Zhang, 2014). In all cases, the current is sinusoidal, and the target can be selected according to the operation mode. For the rectifier operation, the most beneficial for the grid is corresponding asymmetry, whereas for the inverter operation, the most beneficial current is opposite asymmetry. When one of the phase currents has reached the limit, the target may be changed to symmetrical current due to the higher power possible to be transferred with this target (Iwanski, 2019).

Positive- and negative-sequence components' decomposition of signals in different coordinate frames and their separate control paths (Kim et al. 1993; Rioual et al., 1993) was done using proportional integral terms or, in some publications, proportional-integral-resonant controllers in a rotating frame ( $\mathrm{Hu}$ and $\mathrm{He}, 2008)$. Sometimes, current control is realized by proportional-resonant terms in a stationary frame (Guo et al., 2018; Shabestary and Mohamed, 2019; Shuai et al., 2019) or in a natural $a b c$ frame (Guo et al., 2017). The first family of methods with separate control paths for positive and negative sequences causes problems in realization of current limitation methods, which are complicated when an asymmetrical current target is selected. The second family of methods with resonant terms creates problems with anti-wind-up structures of resonant terms, which are not as intuitive and simple as in proportional-integral controllers. 


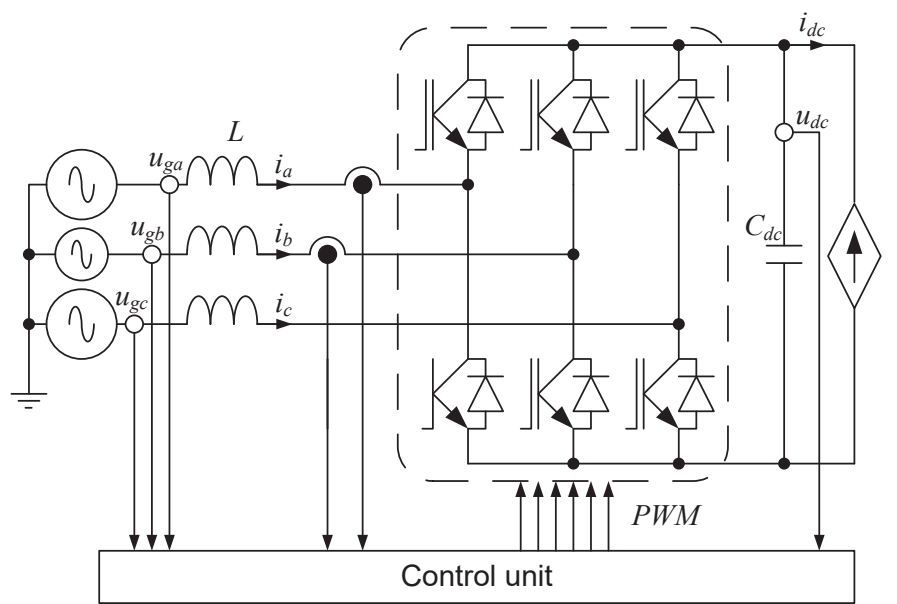

Fig. 1. Scheme of the power circuit of a three-phase converter operating with unbalanced grid voltage.

This paper presents a new control method of a three-phase converter current using transformation to the nonCartesian frame (Iwański et al., 2019), the parameters of which are matched to the voltage asymmetry, and the input and output signals of the current controllers are constant, when current imbalance is referenced. Constant components of controlled current vector and constant output signals of controllers make it possible to resign from oscillatory terms in controller structures, which simplifies current limitation and anti-wind-up structures of controllers, which are reduced only to proportional-integral terms like in the classic voltage-oriented control method.

The paper presents variants of control methods for unbalanced current target with asymmetry corresponding to the voltage asymmetry (oscillating $p$ and constant $q$ components of instantaneous power), symmetrical sinusoidal current target (both $p$ and $q$ oscillating) and unbalanced current target with asymmetry opposite to the voltage asymmetry (constant $p$ component and oscillating $q$ component of instantaneous power). In each target, the transformation parameters are matched adequately to obtain constant current vector components and constant output signals of current controllers.

\section{CONTROL METHODS USING NON-CARTESIAN FRAME TRANSFORMATION}

\subsection{CURRENT ASYMMETRY CORRESPONDING TO THE GRID VOLTAGE ASYMMETRY}

The proposed control method providing three-phase converter current asymmetry corresponding to the grid voltage asymmetry is presented in Fig. 2.

The $\alpha \beta$ to $\alpha^{\prime} \beta^{\prime}$ transformation of the converter current's vector components is as follows:

$$
\left[\begin{array}{c}
i_{\alpha}^{\prime} \\
i_{\beta}^{\prime}
\end{array}\right]=\frac{1}{\sin \left(\theta_{\beta \alpha}^{\prime}\right)}\left[\begin{array}{cc}
M_{\alpha} \sin \left(\theta_{\beta}^{\prime}\right) & -M_{\alpha} \cos \left(\theta_{\beta}^{\prime}\right) \\
-M_{\beta} \sin \left(\theta_{\alpha}^{\prime}\right) & M_{\beta} \cos \left(\theta_{\alpha}^{\prime}\right)
\end{array}\right]\left[\begin{array}{c}
i_{\alpha} \\
i_{\beta}
\end{array}\right]
$$

where

$$
\left[\begin{array}{c}
M_{\alpha} \\
M_{\beta}
\end{array}\right]=\left[\begin{array}{c}
\frac{\left|u_{g a b c}\right|^{\text {max }}}{\left|u_{g \beta}\right|} \\
\frac{\left|u_{g a b c}\right|^{\max }}{\left|u_{g \alpha}\right|}
\end{array}\right]
$$


$\theta_{\alpha}^{\prime}$ is the angle between the $\alpha^{\prime}$ axis of a new frame and the $\alpha$ axis of the natural stationary frame, $\theta_{\beta}^{\prime}$ is the angle between the $\beta^{\prime}$ axis of a new frame and the $\alpha$ axis of the natural stationary frame, $\theta_{\beta \alpha}^{\prime}$ is the angle between the $\beta^{\prime}$ and $\alpha^{\prime}$ axes of a new frame and $\left|u_{\text {gabc }}\right|^{\max }$ in $M_{\alpha}$ and $M_{\beta}$ factors is selected according to the following equation:

$\left|u_{g a b c}\right|^{\max }=\max \left\{\left|u_{g a}\right|,\left|u_{g b}\right|,\left|u_{g c}\right|\right\}$
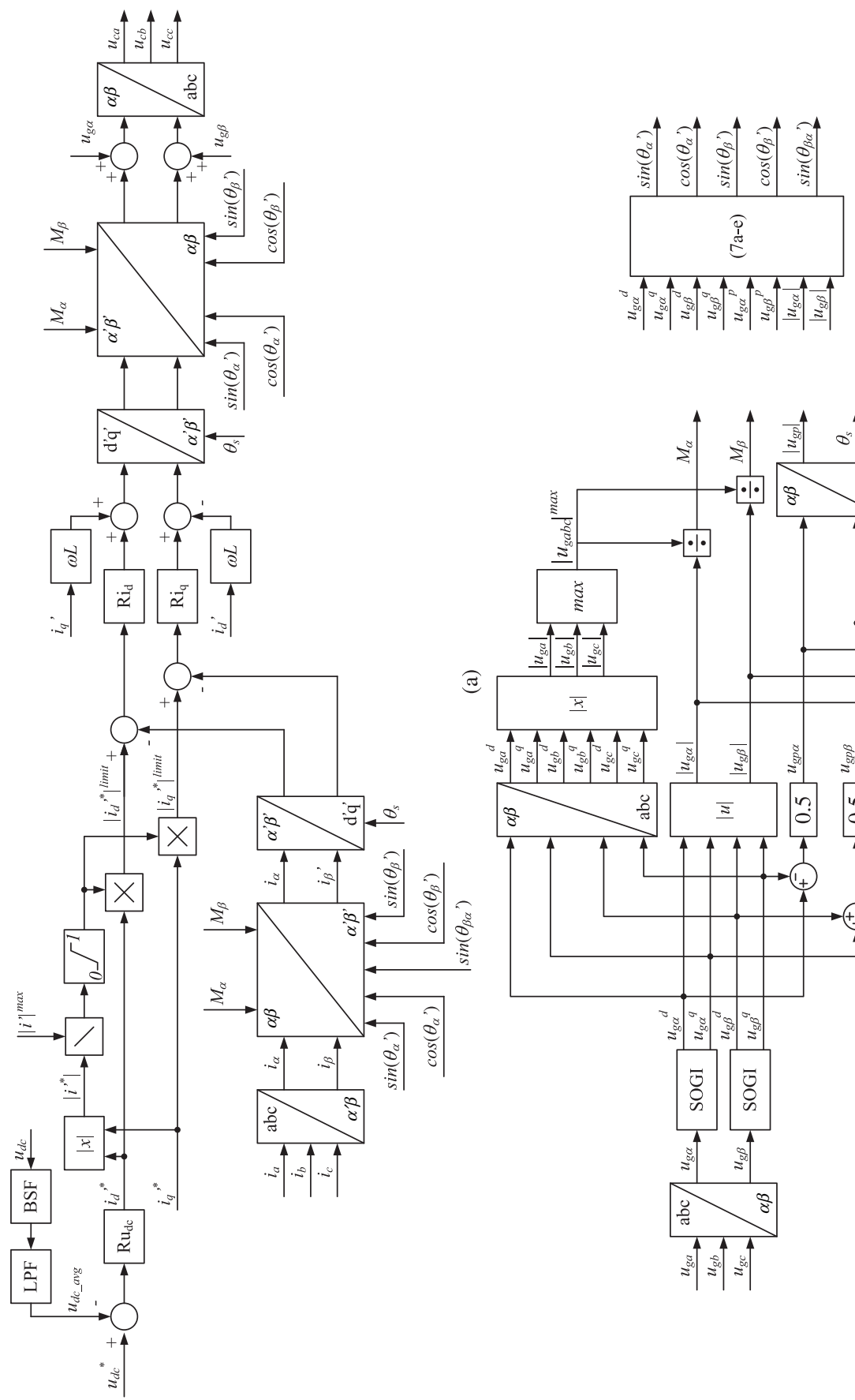

Fig. 2. Proposed control method (a) and calculation of the non-Cartesian frame transformation parameters (b) for unbalanced current target with asymmetry corresponding to the voltage asymmetry. 
Amplitudes of $\left|u_{a}\right|,\left|u_{b}\right|$ and $\left|u_{c}\right|$ phase signals can be assigned using inverse Clarke's transformation for each direct and quadrature phase signal $u_{a}^{d}, u_{b}^{d}$ and $u_{c}^{d}$ and $u_{a}^{q}, u_{b}^{q}$ and $u_{c}^{q}$, respectively.

$$
\begin{gathered}
u_{g a}^{d}=u_{g \alpha}^{d} \\
u_{g b}^{d}=-\frac{1}{2} u_{g \alpha}^{d}+\frac{\sqrt{3}}{2} u_{g \beta}^{d} \\
u_{g c}^{d}=-\frac{1}{2} u_{g \alpha}^{d}-\frac{\sqrt{3}}{2} u_{g \beta}^{d} \\
u_{g a}^{q}=u_{g \alpha}^{q} \\
u_{g b}^{q}=-\frac{1}{2} u_{g \alpha}^{q}+\frac{\sqrt{3}}{2} u_{g \beta}^{q} \\
u_{g c}^{q}=-\frac{1}{2} u_{g \alpha}^{q}-\frac{\sqrt{3}}{2} u_{g \beta}^{q} \\
\left|u_{g a}\right|=\sqrt{\left(u_{g a}^{d}\right)^{2}+\left(u_{g a}^{q}\right)^{2}}=\left|u_{g \alpha}\right| \\
\left|u_{g b}\right|=\sqrt{\left(u_{g b}^{d}\right)^{2}+\left(u_{g b}^{q}\right)^{2}} \\
\left|u_{g c}\right|=\sqrt{\left(u_{g c}^{d}\right)^{2}+\left(u_{g c}^{q}\right)^{2}}
\end{gathered}
$$

This way the amplitudes of $\alpha^{\prime} \beta^{\prime}$ current vector components have the amplitudes equal to the highest amplitude from among amplitudes of phase currents $\left|i_{a}\right|,\left|i_{b}\right|$ and $\left|i_{c}\right|$, because the hodograph of the current vector has the same orientation as the hodograph of the grid voltage vector. It makes easy implementation of reference current limitation possible so as not to exceed converter current constraints. Transformation angles are calculated using direct and quadrature components of $u_{g \alpha}$ and $u_{g \beta}$ as well as positive-sequence grid voltage vector $\alpha \beta$ components $u_{g p a}$ and $u_{g p \beta}$ :

$$
\begin{aligned}
& \sin \left(\theta_{\alpha}^{\prime}\right)=\frac{u_{g p \beta} u_{g \alpha}^{d}-u_{g p \alpha} u_{g \alpha}^{q}}{\left|u_{g \alpha}\right|\left|u_{g p}\right|} \\
& \cos \left(\theta_{\alpha}^{\prime}\right)=\frac{u_{g \alpha}^{d} u_{g p \alpha}+u_{g \alpha}^{q} u_{g p \beta}}{\left|u_{g \alpha}\right|\left|u_{g p}\right|} \\
& \sin \left(\theta^{\prime}{ }_{\beta}\right)=\frac{u_{g p \beta} u_{g \beta}^{d}-u_{g p \alpha} u_{g \beta}^{q}}{\left|u_{g \beta}\right|\left|u_{g p}\right|} \\
& \cos \left(\theta_{\beta}^{\prime}\right)=\frac{u_{g \beta}^{d} u_{g p \alpha}+u_{g \beta}^{q} u_{g p \beta}}{\left|u_{g \beta}\right|\left|u_{g p}\right|} \\
& \sin \left(\theta_{\beta \alpha}^{\prime}\right)=\frac{u_{g \beta}^{d} u_{g \alpha}^{q}-u_{g \alpha}^{d} u_{g \beta}^{q}}{\left|u_{g \alpha}\right|\left|u_{g \beta}\right|}
\end{aligned}
$$




\subsection{SYMMETRICAL SINUSOIDAL CURRENT TARGET}

The control method for symmetrical sinusoidal current target is presented in Fig. 3. Transformation parameters are set arbitrarily constant to eliminate the influence of the $\alpha \beta$ to $\alpha^{\prime} \beta^{\prime}$ transformation on the converter current vector components and $\alpha^{\prime} \beta^{\prime}$ to $\alpha \beta$ transformation on the output signals from the current control loop. However, we can resign from the $\alpha \beta$ to $\alpha^{\prime} \beta^{\prime}$ transformation and opposite $\alpha^{\prime} \beta^{\prime}$ to $\alpha \beta$ transformation at all, modifying the control code.

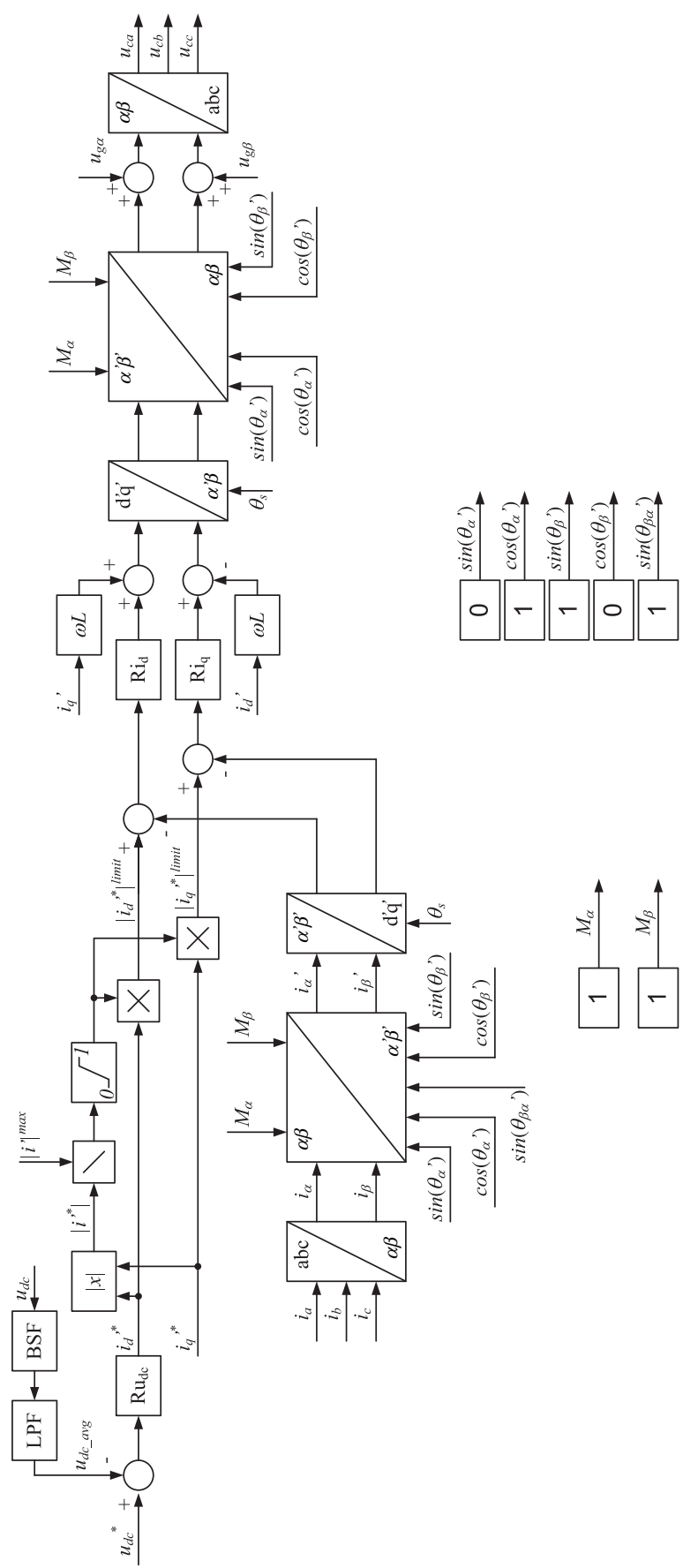

Fig. 3. Proposed control method and arbitrary selection of the non-Cartesian frame transformation parameters for symmetrical sinusoidal current target despite voltage asymmetry. 


\subsection{CURRENT ASYMMETRY OPPOSITE TO THE GRID VOLTAGE ASYMMETRY}

Converter current asymmetry opposite to the grid voltage asymmetry can also be realized using non-Cartesian frame transformation, but first it requires some analysis and re-calculation of transformation parameters to achieve constant current vector components in a new frame despite opposite asymmetry and to achieve true limitation of the unbalanced phase current, that is not to exceed the current maximum rms value in any phase.

Fig. 4 presents the elliptic hodograph of the unbalanced grid voltage vector inscribed in the hexagon. The vector projection in any position does not exceed the hexagon setting the maximum value of the phase voltage, which is a base for scaling factors $M_{\alpha}$ and $M_{\beta}$ of non-Cartesian frame transformation. Opposite orientation of the vector hodograph representing opposite asymmetry causes the vector projection in some positions to exceed the hexagon, which means that the base $\left|u_{\text {gabc }}\right|^{\max }$ must be recalculated by finding the maximum phase voltage $\left|u_{\text {gabc }}^{r}\right|^{\max }$ for oppositely oriented hodographs. The new base $\left|u_{\text {gabc }}^{r}\right|^{\max }$ is calculated based on the new direct and quadrature components of the voltage vector represented in the $\alpha \beta$ frame $u_{g \alpha}^{d r}, u_{g \alpha}^{q r}$ and $u_{g \beta}^{d r}, u_{g \beta}^{q r}$. For opposite orientation of the vector hodographs, the following relations are met:

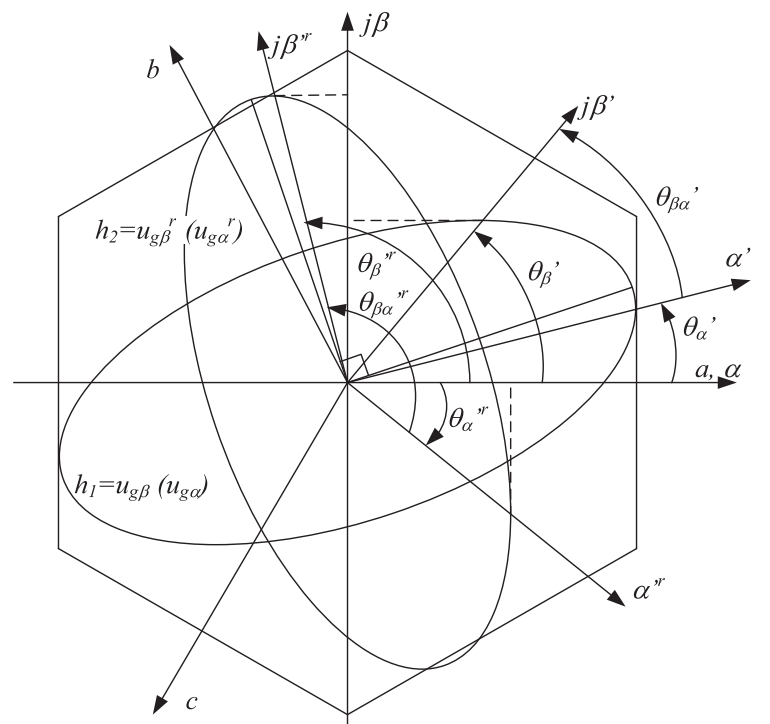

Fig. 4. Graphic presentation of oppositely oriented hodographs of the grid voltage vector during grid voltage asymmetry.

$$
\begin{aligned}
& u_{g \alpha}^{d r}=-u_{g \beta}^{q} \\
& u_{g \alpha}^{q r}=u_{g \beta}^{d} \\
& u_{g \beta}^{d r}=u_{g \alpha}^{q} \\
& u_{g \beta}^{q r}=-u_{g \alpha}^{d}
\end{aligned}
$$

New $a b c$ components of the grid voltage vector corresponding to the oppositely oriented hodograph are calculated according to the following equations:

$$
\begin{gathered}
u_{g a}^{d r}=u_{g \alpha}^{d r} \\
u_{g b}^{d r}=-\frac{1}{2} u_{g \alpha}^{d r}+\frac{\sqrt{3}}{2} u_{g \beta}^{d r} \\
u_{g c}^{d r}=-\frac{1}{2} u_{g \alpha}^{d r}-\frac{\sqrt{3}}{2} u_{g \beta}^{d r}
\end{gathered}
$$




$$
\begin{gathered}
u_{g a}^{q r}=u_{g \alpha}^{q r} \\
u_{g b}^{q r}=-\frac{1}{2} u_{g \alpha}^{q r}+\frac{\sqrt{3}}{2} u_{g \beta}^{q r} \\
u_{g c}^{q r}=-\frac{1}{2} u_{g \alpha}^{q r}-\frac{\sqrt{3}}{2} u_{g \beta}^{q r} \\
\left|u_{g a}^{r}\right|=\sqrt{\left(u_{g a}^{d}\right)^{2}+\left(u_{g a}^{q}\right)^{2}}=\left|u_{g \alpha}^{r}\right| \\
\left|u_{g b}^{r}\right|=\sqrt{\left(u_{g b}^{d}\right)^{2}+\left(u_{g b}^{q}\right)^{2}} \\
\left|u_{g c}^{r}\right|=\sqrt{\left(u_{g c}^{d}\right)^{2}+\left(u_{g c}^{q}\right)^{2}}
\end{gathered}
$$

Finally, the new base voltage is selected according to (12).

$$
\left|u_{g a b c}^{r}\right|^{\max }=\max \left\{\left|u_{g a}^{r}\right|,\left|u_{g b}^{r}\right|,\left|u_{g c}^{r}\right|\right\}
$$

Besides the scaling factors for each axis, adequate angles must be determined because the geometry of the new $\alpha^{\prime r} \beta^{\prime r}$ transformation frame has changed as it is shown in Fig. 4. It can be seen that

$$
\begin{aligned}
& \theta_{\alpha}^{\prime r}=\theta_{\beta}^{\prime}-\frac{\pi}{2} \\
& \theta_{\beta}^{\prime r}=\frac{\pi}{2}+\theta_{\alpha}^{\prime} \\
& \theta_{\beta \alpha}^{\prime r}=\theta_{\beta}^{\prime r}-\theta_{\alpha}^{\prime r}=\frac{\pi}{2}+\theta_{\alpha}^{\prime}-\theta_{\beta}^{\prime}+\frac{\pi}{2}=\theta_{\beta}^{\prime}-\theta_{\alpha}^{\prime}
\end{aligned}
$$

Thus, the trigonometric parameters of transformation can be calculated as

$$
\begin{aligned}
& \sin \left(\theta_{\alpha}^{\prime r}\right)=-\cos \left(\theta_{\beta}^{\prime}\right) \\
& \cos \left(\theta_{\alpha}^{\prime r}\right)=\sin \left(\theta_{\beta}^{\prime}\right) \\
& \sin \left(\theta_{\beta}^{\prime r}\right)=\cos \left(\theta_{\alpha}^{\prime}\right) \\
& \cos \left(\theta_{\beta}^{\prime r}\right)=-\sin \left(\theta_{\alpha}^{\prime}\right) \\
& \sin \left(\theta_{\beta \alpha}^{\prime r}\right)=\sin \left(\theta_{\beta \alpha}^{\prime}\right)
\end{aligned}
$$

This way, in the new $\alpha^{\prime r} \beta^{\prime r}$, thanks to recalculation of transformation parameters, an unbalanced current vector with asymmetry opposite to the grid voltage asymmetry becomes constant, and the vector length in a new frame is exactly equal to the maximum phase current amplitude. It provides, like in case one with corresponding asymmetry, a true phase current limitation of unbalanced current by simple scaling of reference current vector values in a 
new frame. The control method with converter current asymmetry opposite to the grid voltage asymmetry with true-phase current limitation is presented in Fig. 5.
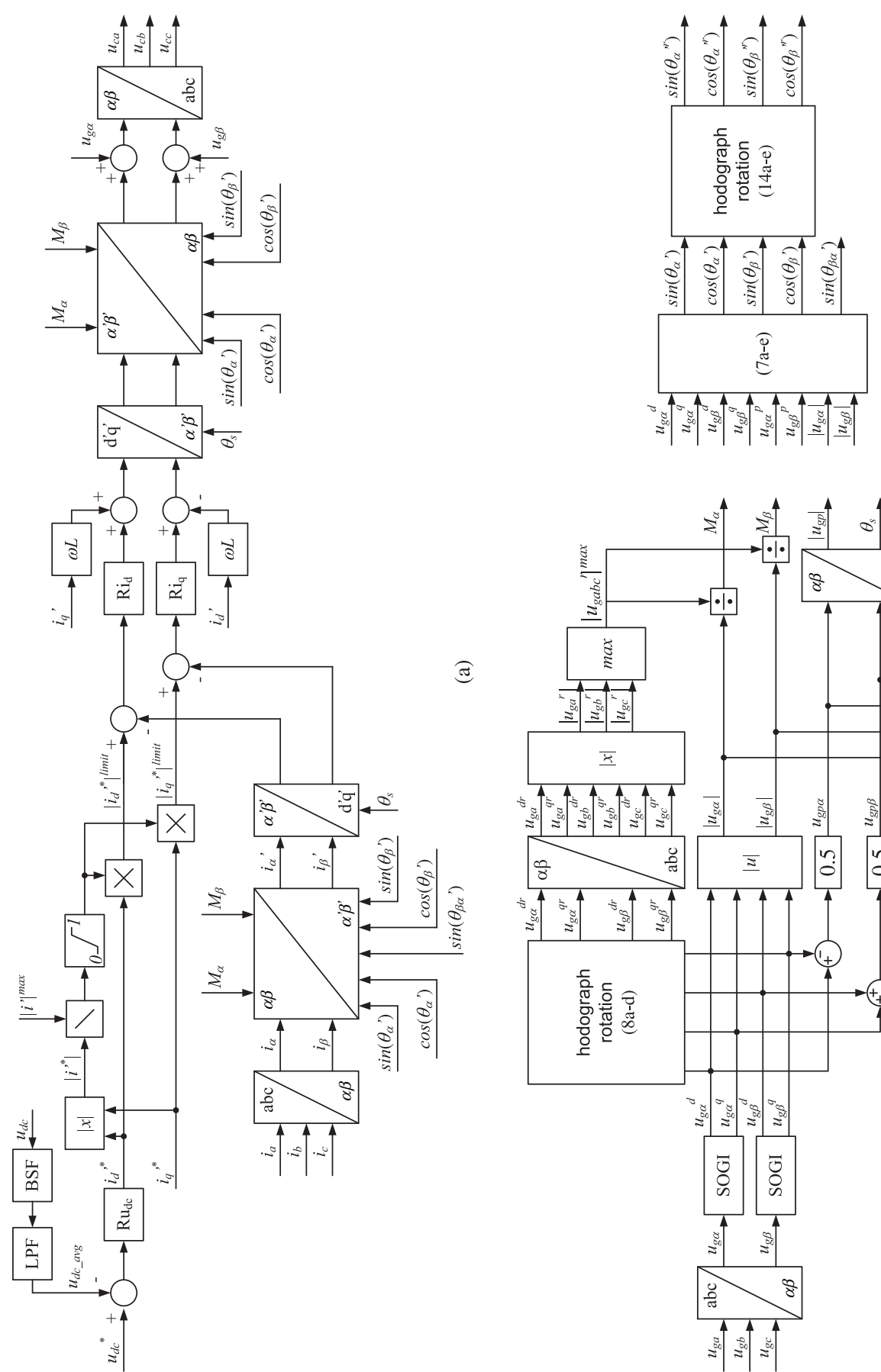

Fig. 5. Proposed control method (a) and calculation of the non-Cartesian frame transformation parameters (b) for unbalanced current target with asymmetry opposite to the voltage asymmetry.

In all presented schemes, the DC voltage controller uses the feedback with filtered DC bus voltage. The set of filters consists of band-stop filter BSF selected with central frequency equal to $100 \mathrm{~Hz}$ to eliminate $100 \mathrm{~Hz}$ oscillations caused by the grid voltage asymmetry and asymmetrical current (oscillating $p$ component of instantaneous power) and low-pass filter LPF with cutoff frequency equal to $150 \mathrm{~Hz}$ to eliminate all high harmonics oscillations produced 


\section{SIMULATION RESULTS OF CONTROL METHODS WITH NON-CARTESIAN FRAME TRANSFORMATION}

Simulation results have been obtained with the following data: $\left|u_{p}\right|=260 \mathrm{~V}$ and $\left|u_{n}\right|=65 \mathrm{~V}$. All tests for each type of reference current asymmetry have been made for the same conditions of grid voltage asymmetry for better comparison. Fig. 6 presents the case of converter current asymmetry corresponding to the grid voltage asymmetry. It can be seen that despite converter current asymmetry, its components in a new rotating $d^{\prime} q^{\prime}$ coordinate frame are constant in steady states. The output signals of the proportional-integral controllers are also constant.

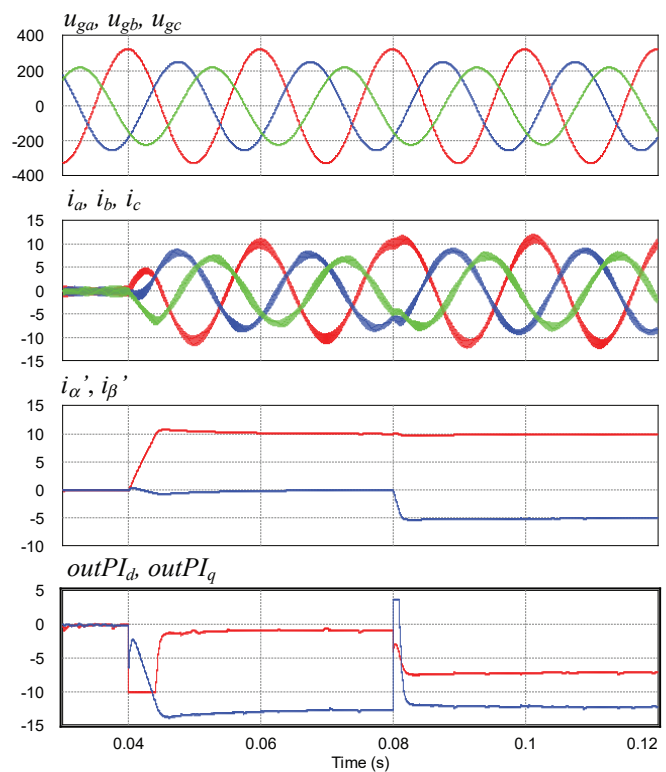

Fig. 6. Simulation results presenting unbalanced grid voltages $u_{g a}, u_{g b}$ and $u_{g c}$; unbalanced converter currents $i_{a}, i_{b}$ and $i_{c}$; converter current vector components in a modified $d^{\prime} q^{\prime}$ rotating frame $i_{d}$ ' and $i_{q}{ }^{\prime}$ and output signals of controllers outPI $I_{d}$ and outPI for current asymmetry corresponding to the voltage asymmetry.

Fig. 7 presents a similar case, but additionally with the occurrence of fifth harmonics in the grid voltage. Owing to the used second order generalized integrator SOGI filtration (Xiao et al., 2017; Xin et al., 2016) in determination of direct and quadrature components of $u_{g \alpha}$ and $u_{g \beta}$ used later for assignment of transformation parameters, the influence of grid voltage harmonics on the converter current harmonics is negligible when the sinusoidal shape of the current is referenced. The harmonics components of the voltage created by the converter needed to achieve sinusoidal current are produced due to relatively high gain of the proportional part of converter current vector component regulators.

Fig. 8 presents the case with symmetrical sinusoidal current target chosen, whereas Fig. 9 presents the case with converter current asymmetry opposite to the grid voltage asymmetry chosen. In both cases, the current vector components and the output signals of current regulators are constant due to adequately matched transformation parameters.

In all cases, the introduced transformations allow to obtain symmetrical sinusoidal current when the grid voltage is symmetrical and with the desired current target at grid voltage imbalance. Fig. 10 presents simulation results of converter operation using asymmetrical grid voltage sag $(25 \%$ of asymmetry calculated as a ratio of negative sequence vector length to the positive sequence vector length) with current asymmetry opposite to the voltage asymmetry during voltage sag and with symmetrical current at symmetrical grid voltage. It can be seen in Fig. 10 


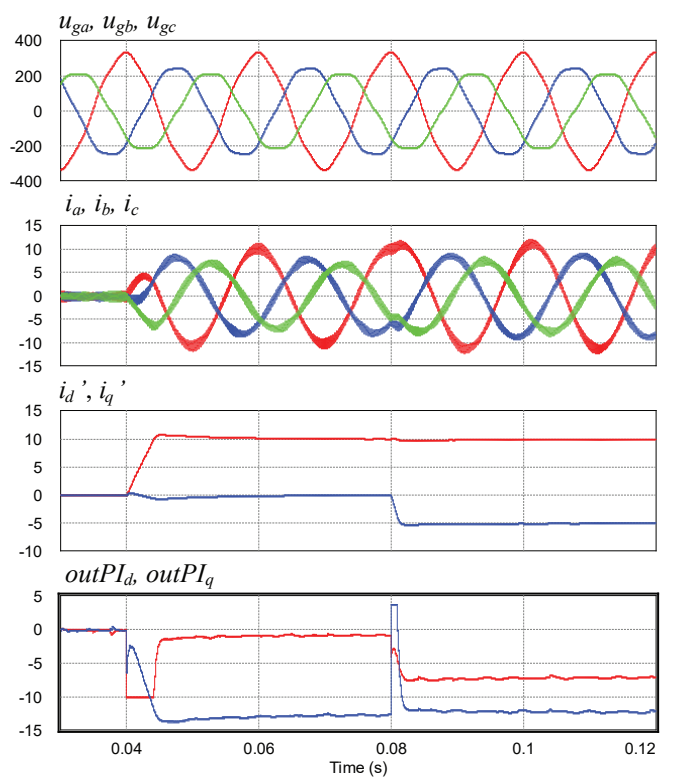

Fig. 7. Simulation results presenting unbalanced grid voltages $u_{g g}, u_{g b}$ and $u_{g c} ;$ unbalanced converter currents $i_{a}, i_{b}$ and $i_{c}$; converter current vector components in a modified $d^{\prime} q^{\prime}$ rotating frame $i_{d}^{\prime}$ and $i_{q}^{\prime}$ and output signals of controllers outPI $I_{d}$ and outPI $I_{q^{\prime}}$ for current asymmetry corresponding to the voltage asymmetry at the occurrence of grid voltage harmonics.

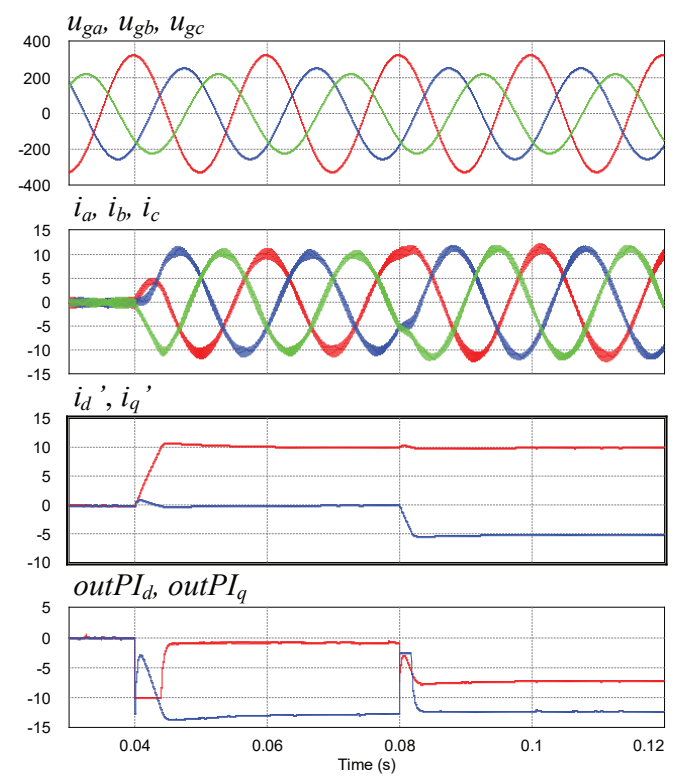

Fig. 8. Simulation results presenting unbalanced grid voltages $u_{g a}, u_{\mathrm{gb}}$ and $u_{\mathrm{c}}$; unbalanced converter currents $i_{a}, i_{b}$ and $i_{c}$; converter current vector components in a modified $d^{\prime} q^{\prime}$ rotating frame $i_{d}^{\prime}$ and $i_{q}^{\prime}$ and output signals of controllers outPI and outPI for symmetrical sinusoidal current target.

that the phase current amplitudes of the converter during sag do not exceed the amplitudes of symmetrical current during symmetrical voltage. Output signals of the current vector components of regulators are disturbed in transient states due to the change in transformation parameters, which are found over a time period. Shortening of this transient may be obtained by more sophisticated and faster filtration than SOGI structures used in this paper. However, faster methods of filtration are more complex, whereas results obtained in the paper, meant as the current quality, are good. 


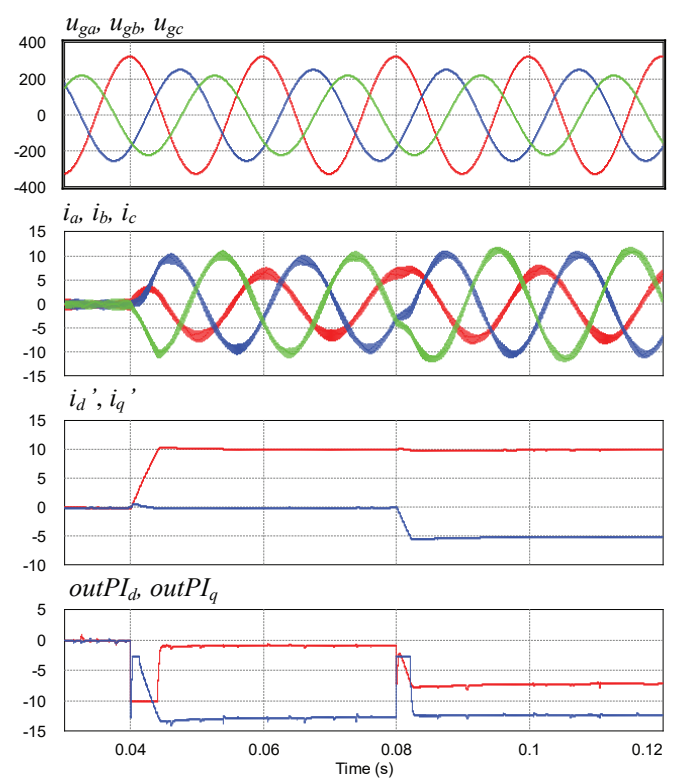

Fig. 9. Simulation results presenting unbalanced grid voltages $u_{g a}, u_{g b}$ and $u_{g c}$; unbalanced converter currents $i_{a}, i_{b}$ and $i_{c}$; converter current vector components in a modified $d^{\prime} q^{\prime}$ rotating frame $i_{d}$ ' and $i_{q}{ }^{\prime}$ and output signals of controllers outPI and outPI for current asymmetry opposite to the voltage asymmetry.

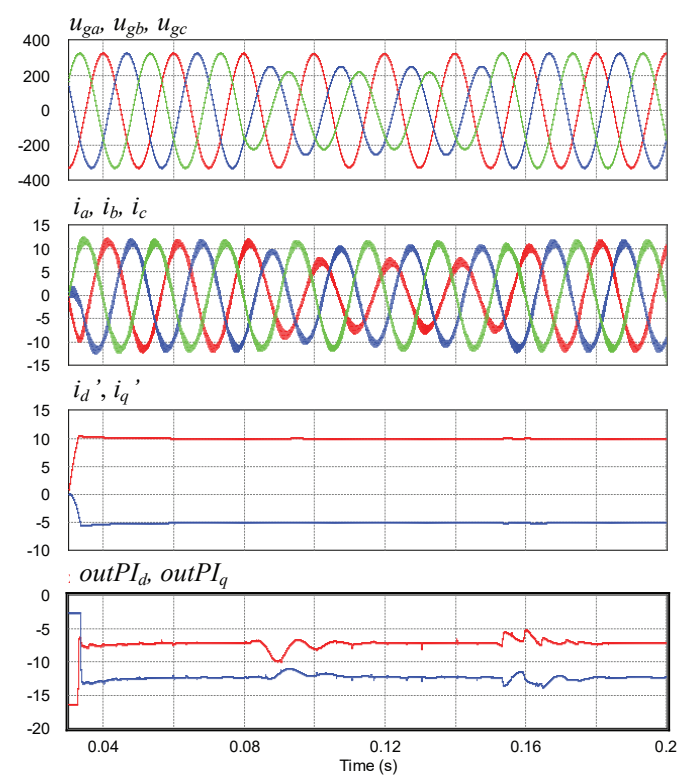

Fig. 10. Simulation results presenting unbalanced grid voltages $u_{s,} u_{s,}$ and $u_{s}$; unbalanced converter currents $i_{,}, i_{b}$ and $i$; converter current vector components in a modified $d^{\prime} q^{\prime}$ rotating frame $i_{d}^{\prime}$ and $i_{q}^{\prime}$ and output signals of controllers outPI $I_{d}$ and outPI for current asymmetry opposite to the voltage asymmetry at $25 \%$ unbalanced grid voltage dip transient.

Simulation tests have been made without $\mathrm{Ru}_{\mathrm{dc}}$ controllers but with arbitrarily referenced current vector components in a modified $d^{\prime} q^{\prime}$ reference frame $\left(i_{d}^{\prime *}\right.$ step from 0 to $10 \mathrm{~A}$ in $0.04 \mathrm{~s}, i_{q}^{\prime *}$ step from 0 to $-5 \mathrm{~A}$ in $\left.0.08 \mathrm{~s}\right)$. Switching frequency equals $10 \mathrm{kHz}, \mathrm{DC}$ bus voltage $-600 \mathrm{~V}$ and filter inductance $-4 \mathrm{mH}$. The operation of control system using the DC voltage controller will be shown in the experimental test results. 


\section{EXPERIMENTAL TESTS OF CONTROL METHODS WITH NON-CARTESIAN FRAME TRANSFORMATION}

Experimental tests have been made with a 5.5-kW power converter, the parameters of which are shown in Table 1. The waveforms of variables have been collected using a multichannel oscilloscope without data processing in any software.

Figs. 11-13 present the power converter operation in the laboratory set-up during grid voltage imbalance and step change in the operating point by increase in the DC bus load or by increase in the power delivered from the DC bus. Fig. 11 presents the case of converter operation with current imbalance corresponding to the grid voltage imbalance. Fig. 12 presents the case with symmetrical current target despite grid voltage imbalance, whereas Fig. 13 presents the case of current imbalance opposite to the grid voltage imbalance. In all cases (Figs. 11-13), after initial no load operation, a 2-kW resistive load and next additional 1.5-kW resistive load during rectifier operation are applied. This is the maximum power that can be obtained in the case of Fig. 11 (current asymmetry corresponding to the voltage asymmetry) without exceeding any sinusoidal phase current constraints (14 A in rms, 20 A in amplitude).

Fig. 14 presents the case of inverter to rectifier change. According to the analysis in Iwanski (2019), the opposite current asymmetry target is most beneficial for the grid of the three presented targets, whereas in the rectifier mode, the corresponding current asymmetry is preferable for the grid. Fig. 15 presents the inverter operation during asymmetrical voltage dip. For symmetrical grid voltage, the converter current becomes symmetrical, whereas during sag, the current has opposite asymmetry. In this test, the DC bus voltage is controlled externally and the current vector components are referenced arbitrarily. It can be seen that during sag, the maximum current amplitude equals the amplitude of balanced current before sag. Fig. 16 presents similar transient but for rectifier operation of converter loaded by $2 \mathrm{~kW}$ resistive load and with enabled DC bus voltage controller. It can be seen that the current amplitude in all phases during voltage sag is higher than before the sag to keep the same amount of power for the resistive load.

Table 1. Parameters of Power Converter Used in the Laboratory Unit

\begin{tabular}{lccc}
\hline Symbol & Parameter & Value & Unit \\
\hline \hline$U_{g n}$ & Nominal phase voltage (L-N rms) & 133 & $\mathrm{~V}$ \\
$I_{n}$ & Rated current rms & 14 & $\mathrm{~A}$ \\
$L$ & Grid filter inductance & 1.2 & $\mathrm{mH}$ \\
$R_{L}$ & Inductor resistance & 40 & $\mathrm{~m} \Omega$ \\
$C_{d c}$ & DC link capacitance & 1 & $\mathrm{mF}$ \\
$f_{s}$ & Switching frequency & 4 & $\mathrm{kHz}$ \\
\hline
\end{tabular}

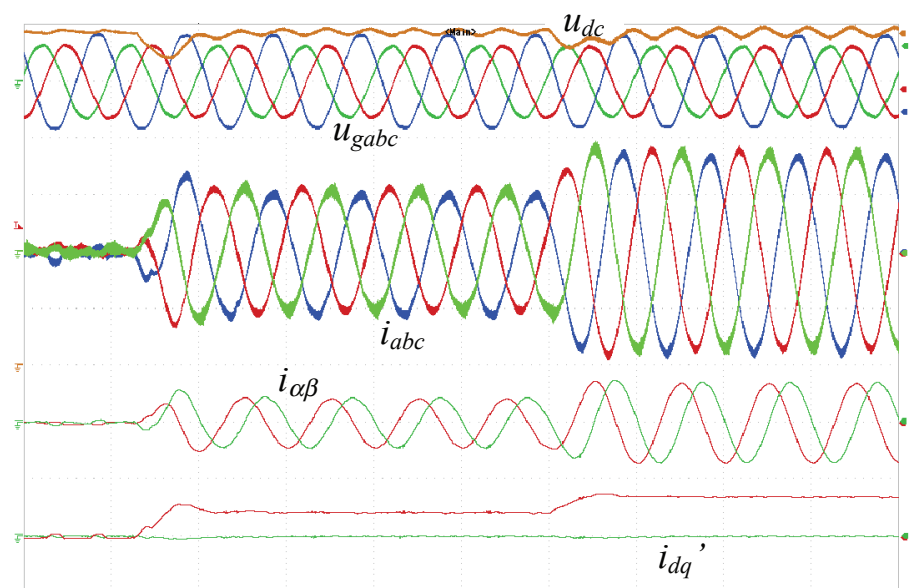

Fig. 11. Experimental test results presenting unbalanced grid voltage $u_{g a b c}$, unbalanced converter current $i_{a b c}$, current components in a natural $\alpha \beta$ frame $i_{\alpha}$ and $i_{\beta}$ and current components in a modified rotating $d^{\prime} q^{\prime}$ frame $i_{d}^{\prime}$ and $i_{q}^{\prime}$ for current asymmetry corresponding to the voltage asymmetry. 


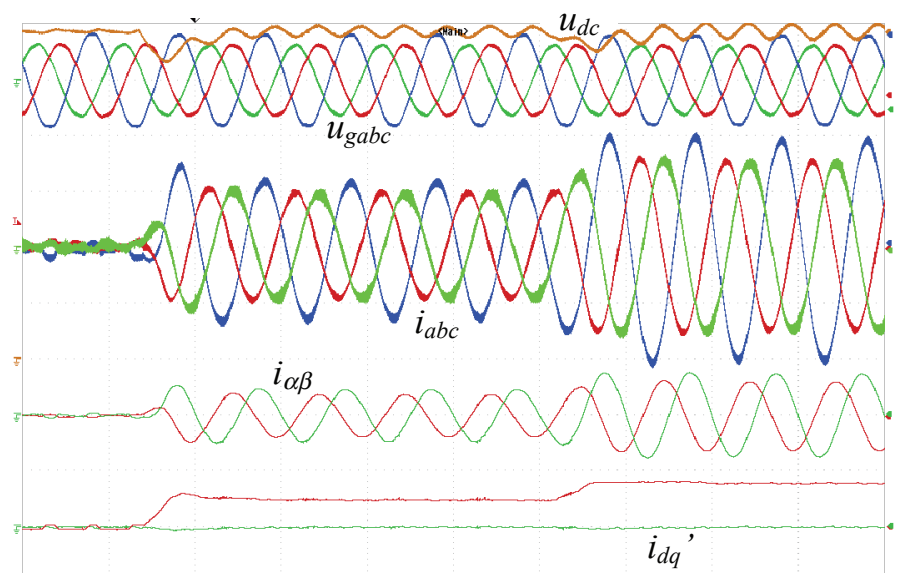

Fig. 12. Experimental test results presenting unbalanced grid voltage $u_{\text {gabc, }}$, unbalanced converter current $i_{a b c}$, current components in a natural $\alpha \beta$ frame $i_{\alpha}$ and $i_{\beta}$ and current components in a modified rotating $d^{\prime} q^{\prime}$ frame $i_{d}^{\prime}$ and $i_{q}^{\prime}$ for symmetrical current target.

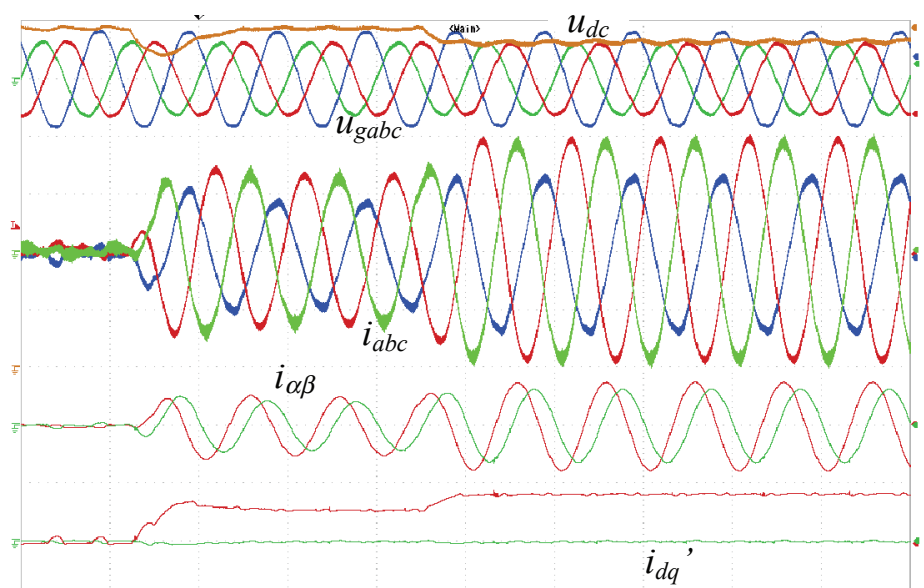

Fig. 13. Experimental test results presenting unbalanced grid voltage $u_{\text {gabc }}$, unbalanced converter current $i_{a b c}$, current components in a natural $\alpha \beta$ frame $i_{\alpha}$ and $i_{\beta}$ and current components in a modified rotating $d^{\prime} q^{\prime}$ frame $i_{d}^{\prime}$ and $i_{q}^{\prime}$ for current asymmetry opposite to the voltage asymmetry.

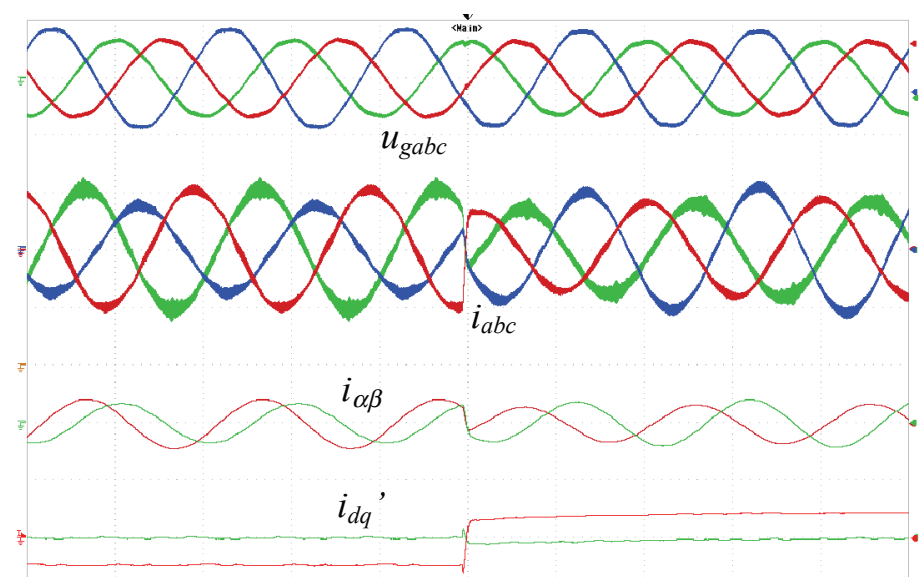

Fig. 14. Experimental tests results presenting unbalanced grid voltage $u_{\text {gabc }}$, unbalanced converter current $i_{a b c}$, current components in a natural $\alpha \beta$ frame $i_{\alpha}$ and $i_{\beta}$ and current components in a modified rotating $d^{\prime} q^{\prime}$ frame $i_{d}^{\prime}$ and $i_{q}^{\prime}$ at the change from inverter mode with opposite current asymmetry to the rectifier mode with corresponding current asymmetry. 


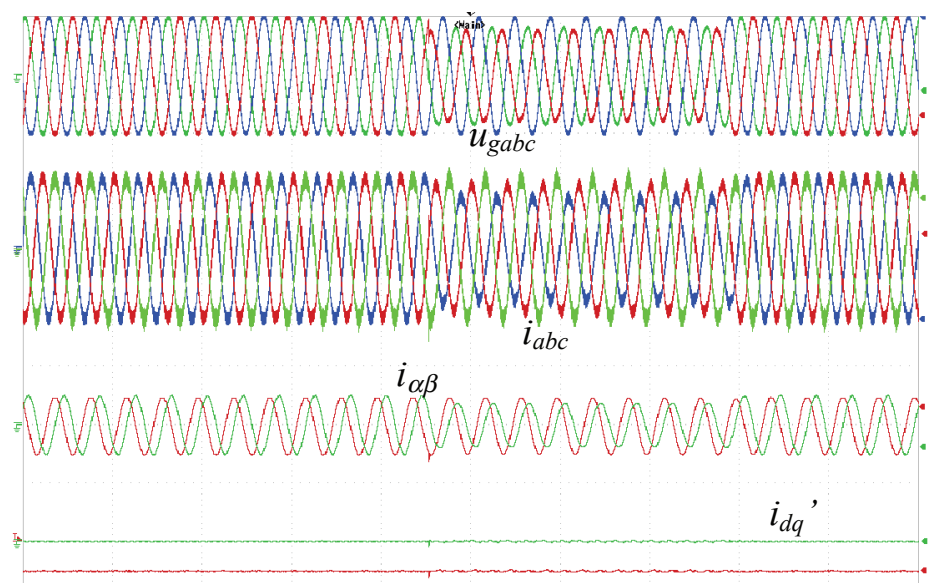

Fig. 15. Experimental test results presenting unbalanced grid voltage $u_{\text {gabc }}$, unbalanced converter current $i_{a b c}$, converter current components in a natural $\alpha \beta$ frame $i_{\alpha}$ and $i_{\beta}$ and converter current components in a modified rotating $d^{\prime} q^{\prime}$ frame $i_{d}^{\prime}$ and $i_{q}^{\prime}$ during an asymmetrical voltage dip in the inverter mode with opposite current asymmetry.

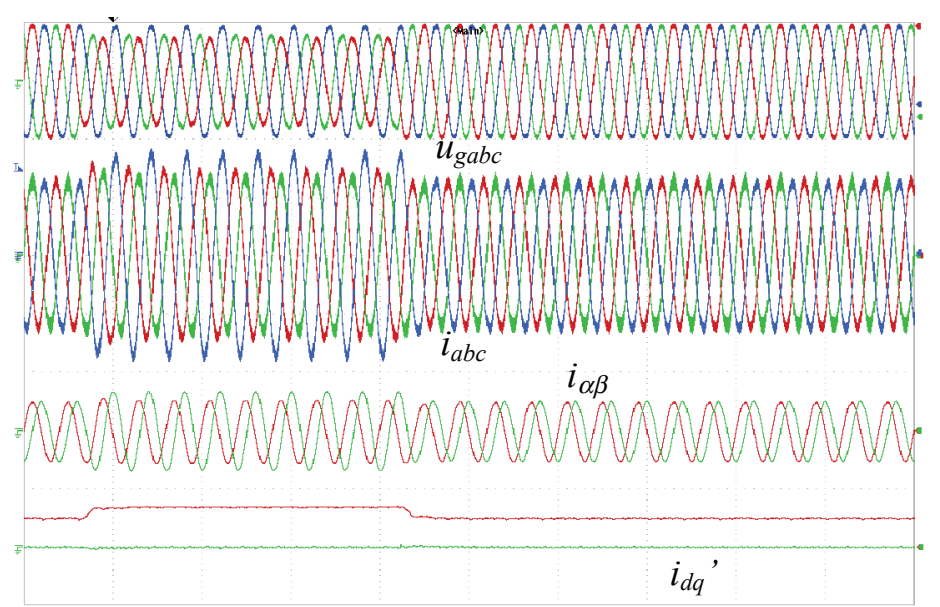

Fig. 16. Experimental test results presenting unbalanced grid voltage $u_{\text {gabc }}$, unbalanced converter current $i_{a b c}$, converter current components in a natural $\alpha \beta$ frame $i_{\alpha}$ and $i_{\beta}$ and converter current components in a modified rotating $d^{\prime} q^{\prime}$ frame $i_{d}^{\prime}$ and $i_{q}^{\prime}$ during an asymmetrical voltage dip in the rectifier mode with corresponding current asymmetry.

\section{CONCLUSION}

The paper presents a control method of a three-phase- three-wire power electronic converter providing separate current targets such as symmetrical sinusoidal current, sinusoidal current having asymmetry corresponding to the grid voltage asymmetry (constant $q$ component of instantaneous power) and sinusoidal current having asymmetry opposite to the grid voltage asymmetry (constant $p$ component of instantaneous power). The methods use a new nonlinear transformation to the stationary frame and then Park's transformation, giving constant components of controlled current despite the reference current imbalance in a natural frame. Adequately matched transformation parameters are calculated without signal decomposition and direct calculation of trigonometric functions. Both simulation results and laboratory test results have proven the correctness of the transformations used and show that the desired current imbalance or balance is obtained. Selected transformation axes scaling factors provide a possibility of true rms current limitations of unbalanced current. 


\section{ACKNOWLEDGEMENTS}

This work was supported by the National Science Centre (Poland) within the project granted on the basis of the decision number 2016/23/B/ST7/03942.

\section{References}

Castilla, M., Mirat, J., Camacho, A., Matas, J. and García de Vicuña, L. (2014). Voltage Support Control Strategies for Static Synchronous Compensators Under Unbalanced Voltage Sags. IEEE Transactions on Industrial Electronics, 61(2), pp. 808-820.

Guo, X., Liu, W. and Lu, Z. (2017). Flexible Power Regulation and Current- Limited Control of GridConnected Inverter Under Unbalanced Grid Voltage Faults. IEEE Transactions on Industrial Electronics, 64(9), pp. 7425-7432.

Guo, X., Yang, Y. and Zhang, X. (2018). Advanced Control of Grid-Connected Current Source Converter Under Unbalanced Grid Voltage Conditions. IEEE Transactions on Industrial Electronics, 65(12), pp. 9225-9233.

Hang, L. and Zhang, M. (2014). Constant Power Control-Based Strategy for Vienna-Type Rectifiers to Expand Operating Area Under Severe Unbalanced Grid. IET Power Electronics, 7(1), pp. 41-49.

$\mathrm{Hu}, \mathrm{J}$. and He, Y. (2008). Modeling and Control of GridConnected Voltage-Sourced Converters Under Generalized Unbalanced Operation Conditions. IEEE Transactions on Energy Conversion, 23(3), pp. 903-913.

Iwanski, G. (2019). Virtual Torque and Power Control of a Three-Phase Converter Connected to an Unbalanced Grid with Consideration of Converter Current Constraint and Operation Mode. IEEE Transactions on Power Electronics, 34(4), pp. 3804-3818.

Iwański, G., Maciejewski, P., Łuszczyk, T. (2019). NonCartesian Frame Transformation based Control of a Three Phase Power Converter During Unbalanced Voltage Dip - Part I: Transformation Principles. Power Electronics and Drives, 4(39).
Kim, H. S., Mok, H. S., Choe, G. H., Hyun, D. S. and Choe, S. Y. (1993). Design of Current Controller for Three-Phase PWM Converter with Unbalanced Input Voltage. In: 29th Annual IEEE Power Electronics Specialists Conference, Fukuoka, Japan, 22-22 May 1993, pp. 503-509.

Rioual, P., Pouliquen, H. and Louis, J. P. (1993). Regulation of a PWM Rectifier in the Unbalanced Network State. In: Proceedings of the 24th Annual IEEE Power Electronics Specialist Conference, Seattle, USA, 20-24 June 1993, pp. 641-647.

Saccomando, G. and Svensson, J. (2001). Transient Operation of Grid-Connected Voltage Source Converter Under Unbalanced Voltage Conditions. 36th IAS Annual Meeting, 4, pp. 2419-2424.

Shabestary, M. M. and Mohamed, Y. A. I. (2019). Asymmetrical Ride-Through and Grid Support in Converter-Interfaced DG Units Under Unbalanced Conditions. IEEE Transactions on Industrial Electronics, 66(2), pp. 1130-1141.

Shuai, Z., Peng, Y., Guerrero, J. M., Li, Y. and Shen, Z. J. (2019). Transient Response Analysis of InverterBased Microgrids Under Unbalanced Conditions Using a Dynamic Phasor Model. IEEE Transactions on Industrial Electronics, 66(4), pp. 2868-2879.

Xiao, F., Dong, L., Li, L. and Liao, X. (2017). AFrequencyFixed SOGl-based PLL for Single-Phase GridConnected Converters. IEEE Transactions on Power Electronics, 32(3), pp. 1713-1719.

Xin, Z., Wang, X., Qin, Z., Lu, M., Loh, P. C. and Blaabjerg, F. (2016). An Improved Second-Order Generalized Integrator Based Quadrature Signal Generator. IEEE Transactions on Power Electronics, 31(12), pp. 8068-8073. 